\title{
Evolution of InAs Quantum Dots during Annealing Process
}

\author{
JiA GuO-ZHI \\ Tianjin Institute of Urban Construction, Tianjin 300384, China \\ YaO Jiang-hong, Shu Yong-Chun, Xin XiaO-Dong \\ AND PI-BIAO \\ The Key Lab of Advanced Technique and Fabrication \\ for Weak-Light Nonlinear Photonics Materials, Ministry of Education \\ TEDA Applied Physics School, Nankai University, Tianjin 300475, China \\ (Received May 5, 2008; revised version July 4, 2008) \\ InAs quantum dots were grown by molecular beam epitaxy in the \\ Stranski-Krastanow growth mode and annealed under $\mathrm{N}_{2}$ atmospheres at \\ different temperatures. The evolution of quantum dots with the anneal- \\ ing temperature increasing were slightly different with the results reported \\ in the literature. Atomic force microscopy investigations of quantum dots \\ uncapped layer show a size initial increase followed by a prompt decrease \\ as annealing temperature increases. It was found that the photolumines- \\ cence signal on quantum dots capped with GaAs layer was first slightly \\ red-shifted and then blue-shifted with an increase in annealing temperature. \\ The blue-shift can be attributed to In/Ga interdiffusion in annealing pro- \\ cess. Red-shift of optical features indicates the change of the quantum dots \\ compostion, size, and strain from the barrier.
}

PACS numbers: 81.16.Dn, 81.05.Ea, 73.21.La

\section{Introduction}

The growth of self-assembled InAs/GaAs quantum dot (QD) structures has been studied extensively in recent years, since these structures offer the prospect of temperature independent, ultralow-threshold lasers. The controllable QDs morphology is crucial for implementing low-dimensional semiconductor device applications [1-3]. Interdiffusion of atoms in semiconductor heterostructures has been used for improving the performance of electronic and optoelectronic devices widely, for example, tuning the emission wavelength of semiconductor device accurately [4]. Previously, the post annealing has been an important controlling and 
manipulating atom composition technique. Annealing process can lead to important changes of shape, composition, structure and strain of QDs, which further influence the spectrum characteristic of QDs. Although effect of the growth conditions on the formation and properties of the QDs has been investigated widely [5-8], effect of annealing on the mobility process and mechanisms of atoms needs to be further investigated, and surface topography evolution and optical properties of QDs.

In this paper, the self-organized InAs QDs without cap layer prepared on $\operatorname{GaAs}(100)$ substrate is analyzed by atomic force microscopy (AFM), and effect of annealing temperature on the evolution of QDs morphology is investigated, and the QDs with cap layer were studied by photoluminescence (PL) under the different annealing conditions. Studies concerning the microstructural and the optical properties of QDs using rapid thermal annealing have been performed to improve the quality of QDs and analysis of the mechanism of QDs evolution. A complete understanding of this evolution process with short annealing period can be used to control the morphology and distribution of QDs.

\section{Experiments}

The InAs QDs are grown on the (100) semi-insulating GaAs wafers in Riber32 compact 21 solid source molecular beam epitaxy (MBE) system equipped with a valved cracker cell for arsenic and reflection high-energy electron diffraction (RHEED) for in situ monitoring of the growth process. Growth temperature is calibrated by infrared pyrometer. After desorbing the surface oxide at $580^{\circ} \mathrm{C}$, a $100 \mathrm{~nm}$ GaAs buffer layer was deposited, then a single layer of QDs was grown by depositing InAs thickness $2.5 \mathrm{ML}$ at $520^{\circ} \mathrm{C}$ growth temperature. The RHEED pattern shows a transformation from streaky to well developed diffraction spots, typical of a three-dimensional (3D) growth mode. During the growth of the subsequent layers (100 $\mathrm{nm}$ GaAs), the RHEED pattern is recovered with streaky pattern and shows a transition from 3D growth mode to two-dimensional (2D) growth mode.

Rapid thermal annealing (RTA) was performed in a SK3-3-10 rapid thermal processor system with a nitrogen ambient and an accurate thermometry measured. The sample was placed between two GaAs wafers without any doping. The annealing temperature is varied from 650 to $800^{\circ} \mathrm{C}$ with a duration $30 \mathrm{~s}$.

The measurements of the distribution and topographic images of uncapped dots were taken ex situ by AFM (SPM Solver P47) with a silicon tip in a working condition of contact mode. PL measurements were carried out directly on this sample with GaAs capping layer. PL was excited using the $532 \mathrm{~nm}$ line of a Nd:YAG laser to evaluate the optical properties of the samples at room temperature.

\section{Results and discussion}

The AFM images taken from samples annealed at different temperature of $650^{\circ} \mathrm{C}, 700^{\circ} \mathrm{C}, 750^{\circ} \mathrm{C}$, and $800^{\circ} \mathrm{C}$ are shown in Fig. 1a-d, respectively. We observed 


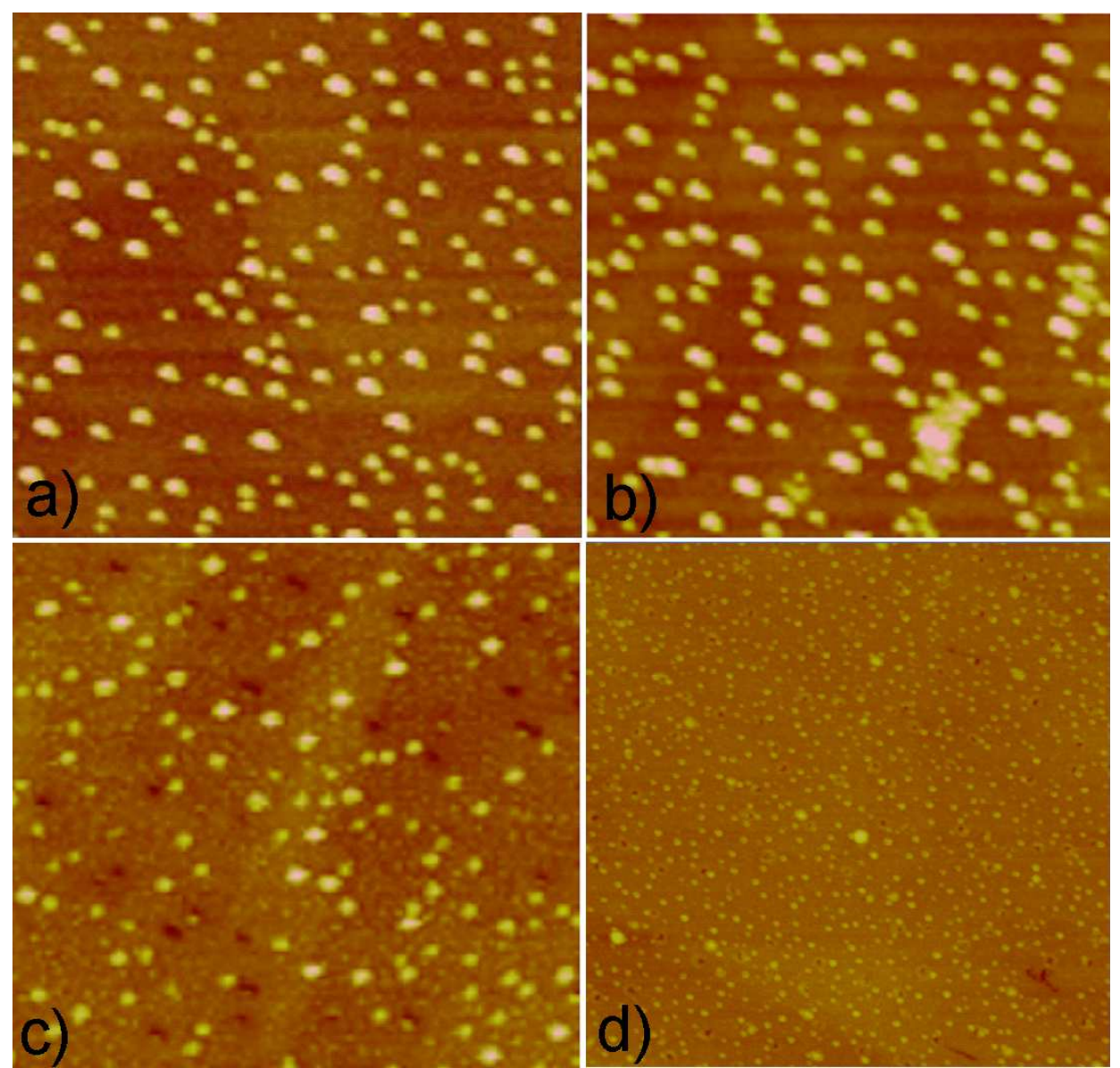

Fig. 1. AFM images $(1 \mu \mathrm{m} \times 1 \mu \mathrm{m})$ of InAs QDs annealed at different temperatures of $650^{\circ} \mathrm{C}(\mathrm{a}), 700^{\circ} \mathrm{C}(\mathrm{b}), 750^{\circ} \mathrm{C}(\mathrm{c}), 800^{\circ} \mathrm{C}(\mathrm{d})$, respectively.

that the height of QDs is bimodal distribution in Fig. 1a and b. The height and lateral distribution of QDs in Fig. 1a is mainly concentrated on near $3.2 \mathrm{~nm}$ and $5.0 \mathrm{~nm}$, the height and lateral distribution in Fig. $1 \mathrm{~b}$ on near $3.9 \mathrm{~nm}$ and $5.2 \mathrm{~nm}$. By comparing Fig. 1a with 1b, we can see that by increasing the annealing temperature, the density of the small dots decreased, while the density of the large dots increases. The height, lateral size, and the density of QDs decrease rapidly, when the annealing temperature was further increased from $700^{\circ} \mathrm{C}$ to $800^{\circ} \mathrm{C}$. According to self-limiting effects of quantum dot, an optimization value at a certain annealing condition exists in strain-induced self-organized growth of QDs $[9,10]$. At the low annealing temperature $\left(T=650^{\circ} \mathrm{C}\right)$, the strain energy between QDs edge and wetting layer will increase with the growth of QDs, which will prevent atoms crossing strain energy barrier to arrive as the quantum dots [11]. The uniform size distribution had not been found for the short annealing period 
due to the atom short diffusion length. When the annealing temperature rises beyond $700^{\circ} \mathrm{C}$, the interdiffusion of In and Ga atoms at the interface between QDs and GaAs matrix can be enhanced, which can result in the strain energy inside the dots significantly reduced. As shown in Fig. 1c, smaller size QDs with lower dot densities are obtained with increasing annealing temperature. It is obvious that the role of atoms migration becomes primary from the wetting layer to QDs surface at lower annealing temperature with the short annealing period ( $t=30 \mathrm{~s}$ ), while the interdiffusion and desorption can be primary at higher annealing temperature. At the same time, the defects can appear in the QDs with large size due to the QDs abnormal regrowing from the fast mass transfer between different size QDs or between QDs and barrier (Fig. 1b).

Figure 2 shows the room temperature PL spectra measured for the samples annealed at different temperature. The low energy peak can be ascribed to the InAs wetting layer. The PL spectrum for the annealed samples shows a dominating peak at near $1210 \mathrm{~nm}$, which is related to interband transitions of InAs QDs. The broadness of PL peaks might origin from the QDs with the various different

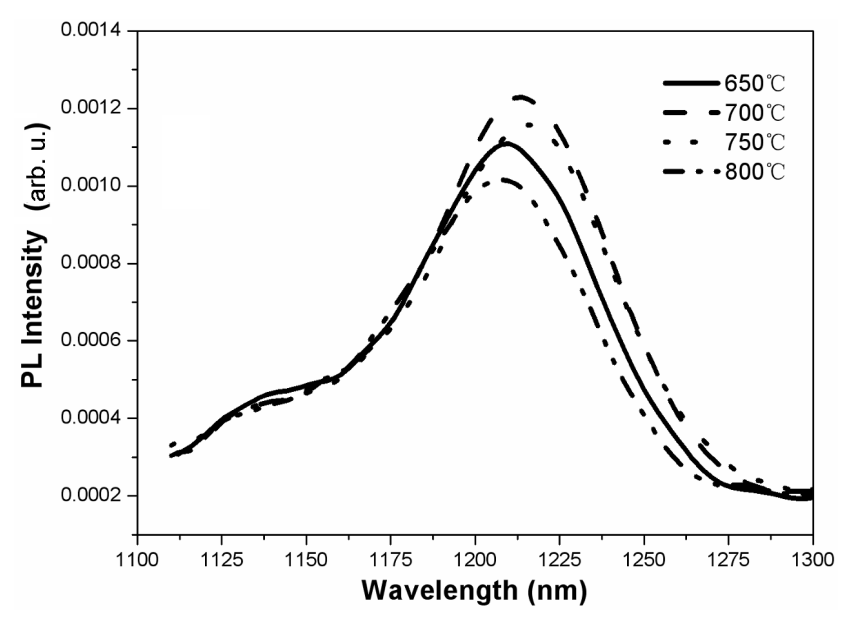

Fig. 2. The PL spectra of the samples with the annealing temperature increase.

sizes. A slight red-shift and blue-shift can be found in the PL peaks at the different annealing process. When the sample was annealed at low temperature, the influence of the annealing process can be weak due to the short diffusion length, which would decrease the effect of composition in QDs on PL peak energy level. It can be confirmed that some of In atom in QDs will diffuse into GaAs cap layer resulting in the strain between QDs and cap layer decreased, which can be the main cause of the red-shift of the QDs sample PL peak [12]. The peak energy and intensity of $\mathrm{PL}$ were changed very little during the annealing process. This also can be ascribed to the diffusion of atom between different size QDs or between QDs and barrier inhibited by the strain from the GaAs cap layer. The results 
cannot simply be ascribed to one of the above mechanisms. The post annealing evolution process of QDs with cap layer is more complicated than that of QDs without cap layer.

\section{Conclusion}

The shape of InAs QDs annealed under nitrogen ambient is investigated at various annealing temperatures. Effect of annealing temperature on the evolution of QDs morphology is investigated by combining the atomic force microscopy and PL spectra. The average size of QDs without cap layer increases first and then decreases with annealing temperature increasing from $650^{\circ} \mathrm{C}$ to $800^{\circ} \mathrm{C}$. A luminescence peak shift of the QDs with GaAs cap layer toward higher energies can result from interdiffusion between barrier and QDs, whose evolution process may be influenced by various mechanisms, including the interdiffusion, and the strain between the GaAs cap layer and InAs QDs and so on.

\section{Acknowledgments}

We thank Xu Bo (Key Laboratory of Semiconductor Materials Science, Institute of Semiconductors, Chinese Academy of Sciences, Beijing) for helpful discussions. This work is financially supported by Science and Technology Plan Projects of the Ministry of Construction of China, the Natural Science Foundation of Tianjin (08JCYBJC14800), The National High Technology Research and Development Program of China (863 Program) (2006AA03Z413), Chinese National Key Basic Research Special Fund (2006CB921703).

\section{References}

[1] H.K. Yong, S.P. Jin, H.L. Uk, C.H. Song, Appl. Phys. Lett. 82, 1099 (2003).

[2] O.B. Shchekin, G. Park, D.L. Huffaker, D.G. Deppe, Appl. Phys. Lett. 77, 466 (2000).

[3] I. Mukhametzhanov, Z. Wei, R. Heitz, A. Madhukar, Appl. Phys. Lett. 75, 85 (1999).

[4] X.C. Wang, S.J. Xu, S.J. Chua, Z.H. Zhang, J. Appl. Phys. 86, 2687 (1999).

[5] H.S. Lee, J.Y. Lee, T.W. Kim, M.D. Kim, J. Appl. Phys. 94, 6354 (2003).

[6] Jin Soo Kim, Jin Hong Lee, Sung Ui Hong, Won Seok Han, Ho-Sang Kwack, Jong Hee Kim, Dae Kon Oh, J. Appl. Phys. 94, 2486 (2003).

[7] E.C. Le Ru, J. Fack, R. Murray, Phys. Rev. B 67, 245318 (2003).

[8] A.O. Kosogov, P. Werner, U. Gösele, N.N. Ledentsov, D. Bimberg, V.M. Ustinov, A.Yu. Egorov, A.E. Zhukov, P.S. Kop'ev, N.A. Bert, Zh.I. Alferov, Appl. Phys. Lett. 69, 3072 (1996).

[9] C.T. Foxon, B.A. Joyce, J. Cryst. Growth. 44, 75 (1978).

[10] D. Leonard, M. Krishnamurthy, S. Fafard, J.L. Merz, P.M. Petroff, J. Vac. Sci. Technol. B 12, 1063 (1994).

[11] Y. Chen, J. Washburn, Phys. Rev. Lett. 77, 4046 (1996).

[12] Jin Soo Kim, Jin Hong Lee, Sung Ui Hong, Won Seok Han, Ho-Sang Kwack, Jong Hee Kim, Dae Kon Oh, J. Appl. Phys. 94, 2486 (2003). 